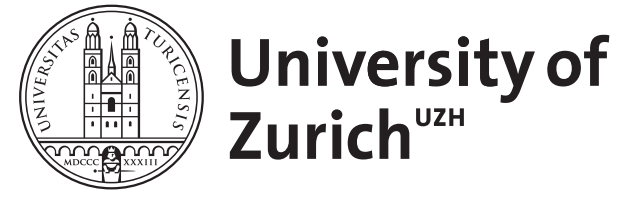

\title{
Venöse Erkrankungen
}

Loo, Bernd ; Lüscher, Thomas F

DOI: https://doi.org/10.1007/s00059-007-2970-7

Posted at the Zurich Open Repository and Archive, University of Zurich ZORA URL: https://doi.org/10.5167/uzh-156662

Journal Article

Published Version

Originally published at:

Loo, Bernd; Lüscher, Thomas F (2007). Venöse Erkrankungen. Herz, 32(1):1-2.

DOI: https://doi.org/10.1007/s00059-007-2970-7 


\title{
Venöse Erkrankungen
}

\author{
Bernd van der Loo, Thomas F. Lüscher ${ }^{1}$
}

${ }^{1}$ Klinik für Kardiologie, Departement Innere Medizin HerzKreislaufZentrum, Universitätsspital Zürich, Schweiz.
Ein Schwerpunktheft über „Venöse Erkrankungen“ in der Zeitschrift Herz ist eine Premiere. Und gewiss, auf den ersten Blick erscheint es möglicherweise etwas überraschend, sich einem solchen Thema in einer Zeitschrift mit kardiologischem Schwerpunkt und entsprechender Leserschaft zu widmen. Im Alltag jedoch werden Kardiologen, sei es in der eigenen Praxis, in vernetzten klinischen Kompetenzzentren der kardiovaskulären Medizin mit entsprechend enger interdisziplinärer Zusammenarbeit mit Angiologen, Radiologen und Chirurgen oder auf internistischen Intensivstationen, sicher regelmäßig mit hier behandelten Fragestellungen und Problemen konfrontiert.

Am offensichtlichsten ist der Zusammenhang natürlich für die venöse Thromboembolie, ist doch die akute Lungenembolie bei älteren hospitalisierten Patienten nach Myokardinfarkt und zerebrovaskulärem Insult die häufigste Erkrankung des Herz-Kreislauf-Systems und auch auf Notfallstationen eine wichtige Differentialdiagnose bei Patienten mit Atemnot und/oder Thoraxschmerzen [1]. Schließlich zeigen verschiedene Untersuchungen, dass auf internistischen Stationen die Thromboembolieprophylaxe - in Gegensatz etwa zu Chirurgen und Frauenärzten - zu wenig eingesetzt wird [2]. Dies weist darauf hin, dass sich in den Fächern der Inneren Medizin nicht in gleicher Weise das Bewusstsein für eine angemessene Thromboembolieprophylaxe entwickelt hat wie in den chirurgischen Fächern, in welchen diese Komplikation nach ope-rativen Eingriffen sehr gefürchtet ist. Umso wichtiger erscheint es, den Schwerpunkt der Zeitschrift Herz diesem Themenbereich zu widmen. Und in der Tat zeigte eine kürzliche Untersuchung mit einem computerisierten Warnsystem für behandelnde Ärzte, dass damit nicht nur die Thromboembolieprophylaxe besser sichergestellt werden kann, sondern auch das Überleben der Patienten günstig beeinflusst wird [3]. Umso wichtiger ist eine gute Fortbildung in diesem Bereich.

Das vorliegende Heft deckt folglich das für den klinischen Alltag relevante Spektrum venöser Erkrankungen $\mathrm{ab}$ und gibt einen hervorragenden Überblick über Physiologie, Pathophysiologie, Klinik, Diagnostik und Therapie von Venenleiden, wobei der Bogen von den potentiell akut lebensbedrohlichen Erkrankungen wie der Lungenembolie bis hin zu den vermeintlichen Schönheitsfehlern gespannt ist.
Hauptfunktion der Venen des Herz-KreislaufSystems ist, wie im Artikel von S. Hochauf \& Schellong, Dresden, über die Funktion des Venensystems dargelegt, zum einen die Speicherung des Blutvolumenanteils, welches aktuell nicht für die Zirkulation benötigt wird, zum anderen der Rücktransport des venösen Bluts zum Herzen [4]. Die Speicherfunktion, d.h. Erweiterung der Venen, wird durch elastische und kollagene Fasernetze in den verschiedenen Schichten der Venenwand gewährleistet. Entscheidende Mechanismen bei der Aufrechterhaltung eines regelrechten Blutrückflusses zum Herzen sind die Venenklappen als spezielle Strukturelemente der Intima sowie die thorakoabdominale Venenpumpe und die periphere Muskelpumpe der unteren Extremitäten.

K. Böhler, Wien, Österreich, behandelt die (rhetorische) Frage, inwieweit die Varikose mehr als nur ein Schönheitsfehler ist [5]. Die Frage ist natürlich vor allem für die Behandlungsnotwendigkeit entscheidend. Während bei Patienten ohne signifikante Zeichen einer venösen Insuffizienz die individuellen Wünsche der Betroffenen noch im Vordergrund stehen, gerade auch bei zunehmender Anwendung neuerer, endoluminaler Therapieverfahren, welche im Beitrag besprochen werden, ist beim Auftreten venöser Ulzera die chirurgische Behandlung einschließlich autologer Spalthautdeckung ein Muss.

Mit der Diagnostik venöser Erkrankungen beschäftigen sich D.D. Do \& M. Husmann, Bern, Schweiz, in ihrem Beitrag [6]. Sowohl bei der Beurteilung des oberflächlichen wie auch des tiefen Venensystems hat sich in den letzten Jahren im klinischen Alltag immer mehr die nichtinvasive Duplexsonographie anstelle der Phlebographie, die gleichwohl für die Diagnostik tiefer Beinvenenthrombosen (TVTs) immer noch „Goldstandard“ bleibt, durchgesetzt. Weitere Vorteile der Duplexsonographie sind das Fehlen von Kontrastmittel- und Strahlenbelastung. Die reine Diagnosestellung ist bei Varikose, Thrombophlebitis sowie der chronischen venösen Insuffizienz oft bereits klinisch gut möglich, die Duplexsonographie liefert zusätzlich exakte morphologische und auch funktionelle Informationen, z. B. zur weiteren Therapieplanung bei Varikose.

Insbesondere in der Diagnostik der TVT ist die Duplex- respektive Kompressionssonographie mit einer Sensitivität von weit über $90 \%$ für die symptomatische proximale TVT und um $90 \%$ für die TVT am Unterschenkel zur Methode der ersten Wahl ge-
Herz 2007;32:1-2 DOI $10.1007 /$ s00059-007-2970-7 
worden - dies umso mehr, wenn die Duplexsonographie mit den Plasma-D-Dimeren sowie klinischen Modellen zur Wahrscheinlichkeitsabschätzung für das Vorliegen einer TVT kombiniert wird [7, 8]. Computertomographie und Magnetresonanztomographie haben vor allem dann Bedeutung, wenn im Becken oder im Abdominalbereich sowohl duplexsonographisch als auch phlebographisch die Diagnose unklar bleibt bzw. um Raumforderungen zu diagnostizieren.

Risikofaktoren, Diagnostik und Therapie der TVT werden von M. Righini \& H. Bounameaux, Genf, Schweiz, ausführlich besprochen [9]. Neben der Akzeptanz der Kompressionsonographie hat das Aufkommen der niedermolekularen Heparine (LMWHs) ganz entscheidend und fast revolutionär im Laufe der letzten 10 Jahre zur grundlegenden Veränderung bei Diagnostik und Therapie der TVT beigetragen. Systemische oder kathetergesteuerte lokale Lysetherapien haben, von ganz wenigen Ausnahmen abgesehen, nur noch historische Bedeutung. LMWHs sind heute zur initialen Standardtherapie der TVT geworden, da sie mindestens genauso effektiv und sicher (Blutungsrisiko, Lungenembolierisiko, Rezidiv einer TVT) wie unfraktioniertes Heparin (UFH) sind, aber die ambulante Behandlung in den allermeisten Fällen ermöglichen und somit klare ökonomische Vorteile bieten. Vor dem Hintergrund der immer weiter zunehmenden „Ökonomisierung“ der meisten Gesundheitssysteme bleibt abzuwarten, inwieweit das Aufkommen von (preiswerterem) subkutan applizierbarem UFH zur ambulanten Behandlung von TVTs in Zukunft mehr als nur eine Alternative zu den LMWHs darstellen wird [10].

Das Auftreten einer Lungenembolie bleibt die gefürchtetste Komplikation einer TVT. Eine asymptomatische Lungenembolie findet sich sogar bei mehr als der Hälfte der Patienten mit symptomatischer proximaler TVT [11]. In ihrem Beitrag beschäftigen sich J. Dörffler-Melly \& B. Amann-Vesti, Zürich, Schweiz, mit der Diagnostik und Therapie der akuten Lungenembolie [12]. In einer im Vergleich zur Diagnostik der TVT nicht unähnlichen Entwicklung hat auch bei der Lungenemboliediagnostik ein nichtinvasives Verfahren (Spiral-CT mit Kontrast) in der klinischen Routine den invasiven „Goldstandard“ (Pulmonalisangiographie) weitgehend ersetzt. Inwieweit bei hämodynamisch stabilen Patienten das Spektrum der aktuellen Therapie der Wahl (UFH oder LMWH oder Fondaparinux mit überlappender Marcumarisierung) durch neuere Antithrombotika erweitert werden wird, bleibt abzuwarten. Bei akuter Lungenembolie und hämodynamischer Instabilität wird in jedem Fall eine schnelle Risikostratifizierung zur Identifizierung der Patienten, welche von einer Thrombolyse oder Embolektomie profitieren, unabdingbar sein [13].

Das Gebiet der venösen Erkrankungen und deren Behandlung wird spannend bleiben. Eine weitere Revolutionierung könnte die Therapie der TVT in Zukunft durch neue synthetische, auch oral applizierbare Substanzen (direkte Thrombin- oder Faktor-X-Inhibitoren) erfahren. Entsprechende Studien sind aktuell im Gange. Ebenso darf man in Zukunft gespannt sein, ob ein substantieller Nutzen (vor allem natürlich im Hinblick auf die Überlebensraten) der kathetertechnischen (oder chirurgischen) Embolektomie sowie der Thrombolyse bei Patienten mit schwerer akuter Lungenembolie gezeigt werden kann.

\section{Literatur}

1. Schellong SM, Bounameaux H, Büller HR. Venous thromboembolism. In: The ESC textbook of cardiovascular medicine. Camm AJ, Lüscher TF, Serruys PW (eds), Blackwell, Oxford, UK, 2006: 1067-92.

2. Chopard P, Dörffler-Melly J, Hess U, Wuillemin WA, Hayoz D, Gallino A, Bächli EM, Canova CR, Isenegger J, Rubino R, Bounameaux $\mathrm{H}$. Venous thromboembolism prophylaxis in acutely ill medical patients: definite need for improvement. $J$ Int Med 2005;257:352-57.

3. Kucher N, Koo S, Quiroz R, Cooper JM, Paterno MD, Soukonnikov B, Goldhaber SZ. Electronic alerts to prevent venous thromboembolism among hospitalized patients. N Engl J Med 2005;352:969-77.

4. Hochauf S, Schellong SM. Struktur und Funktion des venösen Systems. Herz 2007;32:3-7.

5. Böhler K.Varikose: Schönheitsfehler oder Erkrankung? Herz 2007;32:18-25.

6. Do DD, Husmann M. Diagnostik venöser Erkrankungen. Herz 2007;32:10-7.

7. Wells PS, Anderson DR, Rodger M, et al. Evaluation of D-dimer in the diagnosis of suspected deep vein thrombosis. N Engl J Med 2003,349:1227-33.

8. Wells PS, Anderson DR, Bormanis J, et al. Value of assessment of pretest probability of deep-vein thrombosis in clinical management. Lancet 1997;350:1795-8.

9. Righini $M$, Bounameaux $\mathrm{H}$. Venous thrombosis: risk factors and management. Herz 2007;32:27-34.

10. Kearon C, Ginsberg JS, Julian JA, et al. Comparison of fixed-dose weight-adjusted unfractionated heparin and low molecular weight heparin for acute treatment of venous thromboembolism. JAMA 2006;296:935-42.

11. Girard P, Musset D, Parent F, et al. High prevalence of detectable deep venous thrombosis in patients with acute pulmonary embolism. Chest 1999;116:903-8.

12. Dörffler-Melly J, Amann-Vesti B. Diagnostik und Management der akuten Lungenembolie. Herz 2007;32:35-41.

13. Kucher N, Goldhaber SZ. Risk stratification of acute pulmonary embolism. Semin Thromb Hemost 2006;32:838-47.. 